\title{
The Frequency of Positive Cases of Fecal Calprotectin, and Related Factors in Patients with Systemic Sclerosis
}

\section{Alireza Fatemi}

Department of Radiology, School of Medicine, 5th Azar Hospital, Gorgan, Golestan, Iran. Golestan University of Medical Sciences, Gorgan, Golestan, Iran

\section{Reza Zahedpasha}

Department of Radiology, School of Medicine, 5th Azar Hospital, Gorgan, Golestan, Iran. Golestan University of Medical Sciences, Gorgan, Golestan, Iran

\section{Hale Hedayatmofidi}

Rheumatology Research Center, Sayyad Shirazi Hospital, Golestan University of Medical Sciences, Gorgan, Golestan,

\section{Nafiseh Abdolahi ( $\sim$ N_abdolahi2002@yahoo.com )}

Rheumatology Research Center, Sayyad Shirazi Hospital, Golestan University of Medical Sciences, Gorgan, Golestan,

\section{Gharib Hadi}

Department of Radiology, School of Medicine, 5th Azar Hospital, Gorgan, Golestan, Iran. Golestan University of Medical Sciences, Gorgan, Golestan, Iran

\section{Somayeh Livani}

Clinical Research Development Unit (CRDU), Sayad Shirazi Hospital, Golestan University of Medical Sciences, Gorgan, Iran.

\section{Mehrdad Aghaie}

Rheumatology Research Center, Sayyad Shirazi Hospital, Golestan University of Medical Sciences, Gorgan, Golestan,

\section{Research Article}

Keywords: Systemic sclerosis, Fetal calprotectin

Posted Date: March 1st, 2022

DOI: https://doi.org/10.21203/rs.3.rs-1238221/v1

License: (c) (i) This work is licensed under a Creative Commons Attribution 4.0 International License. Read Full License 


\section{Abstract}

Background and aim: SSc (Systemic sclerosis) is a systemic inflammatory heterogeneous disease, characterized by fibrosis of skin and internal organs particularly the gastrointestinal tract by affecting any part of it. Calprotectin is a calcium- and zinc-binding protein and its level increases during an inflammatory process in the presence of neutrophils, measurable in plasma, urine, saliva, and feces. FC (Fecal calprotectin) is a non-invasive and repeatable marker for the diagnosis of intestinal inflammation and unlike other inflammatory markers, the FC level is not affected by other sources of inflammation other than intestines. The quality of life in SSc patients is severely affected by gastrointestinal disorders. So, we decided to examine FC as a simple and accessible method with no complication to diagnose gastrointestinal disorders and the extent of disease activity.

Materials and methods: Totally 46 patients with SSc were invited to include in our study. Seven patients did not enter our study because of having the items in exclusion criteria. For the remaining 39 patients, a questionnaire about gastrointestinal disorders symptoms (UCLA SCTS 2.0) was filled in and then their skin was examined and the skin score was determined. Stool samples were collected to measure the calprotectin level. Barium Swallow and CT Enterography was done to determine gastrointestinal disorders. FC level wasn't less than $50 \mu \mathrm{gr} / \mathrm{gr}$ in any of our cases, therefore the patients was divided into two groups: the one with FC level less than $200 \mu \mathrm{gr} / \mathrm{gr}$ and the one with FC level as $200 \mu \mathrm{gr} / \mathrm{gr}$ or more than $200 \mu \mathrm{gr} / \mathrm{gr}$ and the latter was considered as positive. Chi-Square test was used and to determine the correlation between FC level and age, disease type, qualitative findings in radiography and quantitative variables like skin score.

Results: The correlation between FC level and sex was not verifiable cause all patients were female. Increased levels of FC didn't have any correlation with age ( $P$ Value $=0.79)$, disease type ( $P$ Value $=0.59)$, skin score $(P$ Value $=0.25)$, final $G I$ score $(P$ Value $=0.30)$, esophageal dilatation $(P$ Value $=0.18)$, small intestine wall thickening ( $P$ Value=0.10), and none of serum tests. Also, no signs of micronutrient deficiencies related to FC level was found due to vitamin and mineral supplements consumption in most of our patients.

Discussion and Conclusion: Although there was no statistical correlation between FC level and variables, but the results are in favor of FC specificity to assess intestinal wall thickness, which due to the small sample size requires to be assessed in future researchers' study with a larger sample size.

The main cause of the lack of statistical significance in the relationships of our study can be the low sample size. Therefore, it is recommended that in future researchers' study with a larger sample size.

\section{Introduction}

Systemic sclerosis (SSc, also known as scleroderma) is a systemic inflammatory heterogeneous disease, characterized by fibrosis of skin and internal organs particularly the gastrointestinal tract, where lesions may lead to motor activity impairment, as well as vascular dysfunction and autoimmunity (1-3). This 
collection of seemingly disparate yet interlinked features differentiate SSc from other connective tissue diseases and organ-specific fibrosing disorders (4). According to the extent of skin fibrosis, SSc is divided into three subgroups: diffused, limited and overlap syndrome $(2,5,6)$.

In overlap syndrome, patients have other symptoms of connective tissue diseases, such as Lupus or Myositis, in addition to SSc symptoms (7). Systemic sclerosis is seen all around the world, and it is more frequent in women than in men. The prevalence of SSc varies worldwide and in America it is 250 cases per 1.000 .000 people $(8,9)$. Although SSc is inherited, it is not inherited in Mendelian type (4).

Basically, the disease most often occurs in families with positive history of SSc (1.60\%), relative to the general population (0.026\%); therefore, positive family history is the most important risk factor of SSc yet discovered (10). Gastrointestinal (GI) system is the most involved organ in SSc followed by skin (6); more than 90 percent of SSc patients are reported to have GI manifestations $(1,6,11-13)$. Although, the $G I$ disorders are not the main cause of death in patients, they significantly decrease well-being and quality of life in patients $(6,14)$. Any section of the GI tract could be affected by SSc; however, esophagus is the most frequently involved $(2,15)$; reflux is one of the manifestations which is treated with strong antiacids. Changes in neuromuscular system with progressive fibrosis of smooth muscle in the muscularis propria layer impair normal motor function, which may secondarily affect transit and nutrient absorption $(6,16)$. Anorectal problems are also seen in SSc patients (17-19). Gastric malfunction may lead to different problems such as bleeding as a result of antral vasodilation or delayed gastric emptying (1719); up to $50 \%$ of patients have gastroparesis (6). Bleeding due to dilatation of small bowel, malabsorption syndrome, intestinal pseudo-obstruction and progressive nutritional deficiency are severe complications attributed to intestinal involvement $(6,17-19)$. Pseudo-obstruction syndrome is mainly occurring as a result of small intestinal bacterial overgrowth (SIBO) and it has bad consequences (1820). In addition to fibrosis and motor disorders, inflammation is known to be one of the major factors in development of GI disorders (21). Essentially, the severity of skin fibrosis and GI involvement are not correlated (6).

Calprotectin is a $36-\mathrm{kDa}$ calcium- and zinc-binding protein which constitutes a major ( 40 to $60 \%$ ) part of the cytosolic proteins in neutrophils; it is also found in other myeloid cells and includes a heavy chain of $14 \mathrm{kDa}$ and a light chain of $8 \mathrm{kDa}(22)$. It has a well-known antimicrobial activity and it is probably involved in the regulation of inflammatory reactions. Calprotectin is resistant to enzymatic degradation both in vitro and in vivo and can be easily measured in the stools $(11,23,24)$. In addition, the S100A9 component of the calprotectin compound is a fibroblast growth stimulant (2). During an inflammatory process and in the presence of neutrophils, the level of calprotectin is increased and measurable in the plasma, urine, saliva and stool $(25,26)$. The amount of calprotectin in the stool is specifically linked to the migration of neutrophils to the gastrointestinal tract (27). Therefore, FC is a good marker for the diagnosis of intestinal inflammation (28) and the FC level, unlike other inflammatory markers, such as Erythrocyte sedimentation rate (ESR) and reactive protein C (CRP), is not affected by other sources of inflammation other than intestines (29). During recent years, some researchers have reported high levels of FC in SSc patients $(2,11)$. Fecal calprotectin can be tested and determined by having a small amount 
of stool sample ( $5 \mathrm{~g}$ ) in a buffering solution (24). The FC structure is stable for a week at room temperature $(30,31)$. FC measurement is a non-invasive method and does not require much patient's cooperation. This test is repeatable and its level is clearly elevated in patients with IBD (1). Fecal calprotectin can predict the severity of the disease as well as its recurrence and, moreover, it can be a good measure for evaluating the response to treatment (29). FC diagnostic values for gastrointestinal inflammation are: Values less than $50 \mu \mathrm{g} / \mathrm{g}$ are normal, the values between $100-50 \mu \mathrm{g} / \mathrm{g}$ are considered to be relatively positive and values more than $100 \mu \mathrm{g} / \mathrm{g}$ indicate that the test is positive $(29,32)$ And values more than $200 \mu \mathrm{g} / \mathrm{g}$ are considered to be highly elevated (1). By reviewing the present articles, glucose $\mathrm{H} 2 / \mathrm{CH} 4$ breath test, fecal and serum calprotectin measurement, digestive symptoms questionnaire, Global Symptom Score (GSS), Cineradiography and biochemical tests have been used to diagnose gastrointestinal disorders in SSc patients so far $(1,2,5,6,11)$. Barium sulfate (BaSO4), despite the rare and limited complications, is not harmful to the intestinal mucosa, so this method is widely used in gastrointestinal imaging (33). CT enterography is also a quick and safe method for intestinal imaging (34). Due to the lack of equipment for performing glucose $\mathrm{H} 2$ / $\mathrm{CH} 4$ breath test in most areas and also the high probability of poor patient cooperation to do the test, in this study, we decided to use Barium Swallow and CT-enterography methods and serum micronutrient testing and digestive symptoms questionnaire rather than using glucose $\mathrm{H} 2$ / $\mathrm{CH} 4$ breath test to determine gastrointestinal disorders in SSc patients. To the best of our knowledge, GI disorders are very common in patients with SSc and their complications are greatly annoying.

To date, endoscopy has been the best diagnostic tool for these disorders which gives us not so much information about the extent of disease activity. In addition, the endoscopy process is time consuming, painful and expensive and so it is not pleasant for the patient to repeat it and, on the other hand, small intestine is not accessible by using endoscopy. Therefore, within the current study we decided to use fecal calprotectin measurement as a simple, accessible, non-invasive, painless and repeatable method in order to diagnose gastrointestinal disorders and the extent of disease activity in these patients. Our main goal was the determination of the prevalence of fecal calprotectin positive cases and its related factors in systemic sclerosis patients. Other goals were determination of the prevalence of FC cases in patients with systemic sclerosis by age, sex, disease type, skin score, gastrointestinal symptoms score, small bowel wall thickness in CT-Enterography, esophageal dilation in barium swallow examination and results of blood serum biochemical tests.

\section{Materials And Methods}

\section{Patients}

The study was conducted at the rheumatology department of Sayyad Shirazi hospital (Gorgan, Golestan Province, Iran). The study was approved by the regional ethical board in Golestan University of Medical Sciences, and all patients provided written informed consent. 
Our patients were those with SSc who were visited in the offices of rheumatologists, Rheumatology Clinic in Sayyad Shirazi hospital, patients admitted to the rheumatology department of this hospital and the ones who had gone to Dezyani Hospital Professional Clinic in Gorgan. Our inclusion criteria were decided to be suffering from SSc as a result of the ACR criteria (35) and our exclusion criteria were having other connective tissue diseases or vasculitis, history of hepatic or GI diseases (IBD, diverticulitis and malignancies), diabetes or being alcoholic, history of GI surgery or vagotomy and a history of taking nonsteroidal anti-inflammatory drugs (NSAIDs). A total number of 39 patients, having the previously described circumstances were included in our study.

Considering the median age of 42 years, patients were divided into two groups: the young (less than 42 years) and the old (equal or more than 42 years).

\section{Clinical Assessment:}

The following patient data were collected: age, sex, disease type, skin score, GI symptoms score, small bowel wall thickness in CT-Enterography, esophageal dilation in barium swallow examination and results of blood serum biochemical tests. Patients were classified as having either diffuse cutaneous SSc or limited cutaneous SSc (7). Skin involvement was assessed using the modified Rodnan skin score (mRSS) (36). According to EUSTAR Systemic Sclerosis Activity Score, a skin scores above 14 was considered as a sign of active skin involvement and a score of less than 14 was considered as a sign of inactive skin involvement (37). Esophageal function was assessed by barium swallow and small bowel wall thickness was assessed by CT-Enterography and both were evaluated by a radiologist as normal or involved.

\section{Assessment of medical records}

Medical records were studied carefully and patients with any of the exclusion criteria did not enter the study.

\section{Questionnaire}

Patients were asked to complete the UCLA SCTC 2.0 (University of California Los Angeles Scleroderma Clinical Trial Consortium Gastrointestinal Tract 2.0) (38). This questionnaire was designed specifically for the evaluation of gastrointestinal symptoms in patients with systemic sclerosis and consists of 7 parts: Reflux, bloating/abdominal distension, fecal soilage, diarrhea, social function, emotional well-being and constipation; and each of these topics had some specific questions. The score for each of the questions was calculated individually and at the end, their total was divided into six (Constipation score was not included in the final part) and was considered as the final score. Each patient received a score between zero and 2.83 .

\section{Laboratory examinations}

Determination of FC level was performed by ELISA method using the BÜHLMANN fCAL® EK-CAL (96 test) kit. According to the existing classifications, FC levels above $50 \mu \mathrm{g} / \mathrm{g}$ were considered as increased values $(24,29)$ and FC levels above $200 \mu \mathrm{g} / \mathrm{g}$ were considered to be very highly elevated $(39)$. After 
reaching the $\mathrm{FC}$ levels and considering that this variable was not negative in any patient (less than $50 \mu \mathrm{g} / \mathrm{g}$ ), we decided to classify all patients into two groups, based on their FC level: elevated (greater than $50 \mu \mathrm{g} / \mathrm{g}$ and less than $200 \mu \mathrm{g} / \mathrm{g}$ ) and significantly elevated (greater than $200 \mu \mathrm{g} / \mathrm{g}$ ), therefore, considering the first group as a positive result, and the second group as a negative result.

In all patients, laboratory tests included measurements of Albumin, Magnesium, Zinc, erythrocyte sedimentation rate (ESR), C-reactive protein (CRP), Folic Acid, Vitamin B12 and Ferritin.

\section{Statistical analyses}

According to the literature review, the prevalence of FC positive cases in patients with SSc was 76.5\% (2). Considering the confidence level of 0.95 and accuracy of 0.14 , using the following formula, the sample size was 36 in this study. Finally, considering the approximately 10 percent drop in samples, the final sample size was 40 .

$$
\frac{Z_{1-\alpha / 2}^{2} P(1-P)}{d^{2}}
$$

Collected data were analyzed using Statistical Package for Social Sciences (SPSS) software version 16. Chi-square test was used to determine the relationship between $\mathrm{FC}$ level, age, disease type and qualitative findings in radiography. T-student test was used for the correlation of FC level with quantitative variables such as skin score and GI symptoms, magnesium and ESR, which had normal distribution and MannWhitney test was used for other serum tests with abnormal distribution (CRP, Albumin, Ferritin, folic acid, Vitamin B12 and zinc). A two-tailed P-value $<0.05$ was considered significant.

\section{Results}

A total of 46 patients came to the Sayyad Shirazi Hospital in Gorgan, on certain days but 7 patients, including 2 men and 5 women, didn't enter the study due to exclusion criteria ( 5 cases of diabetes, 1 with history of esophagectomy and 1 with diabetes and poly myositis simultaneously); Therefore, 39 remaining patients in the study were all female. The mean age of the subjects was 43.23 years with a standard deviation of 11.75 years. Table 1 shows the frequency of disease type in the studied population. According to this table, 22 (59.5\%) patients were affected by the diffused type and 15 patients (40.5\%) had limited form of disease. two patients weren't included in the study because of lack of sufficient information to diagnose the type of disease. 
Table 1

Frequency distribution of the disease in the subjects

\begin{tabular}{|lll|}
\hline Disease type & Number & Percentage \\
\hline Diffuse & 22 & 59.50 \\
\hline Limited & 15 & 40.50 \\
\hline Total & 37 & 100 \\
\hline * Unspecified cases were removed. \\
\hline
\end{tabular}

Table 2 shows the distribution of active cases of skin involvement in the subjects. According to this table, 19 people $(57.57 \%)$ had active skin involvement ( 14 s skin score) and 14 patients $(42.43 \%)$ had no active skin involvement (14 > skin score) (37). There was not enough information on the skin score of 6 patients.

Table 2

Frequency distribution of positive cases of skin involvement activity in the subjects

\begin{tabular}{|lll|}
\hline Skin involvement & Number & Percentage \\
\hline Positive & 19 & 57.57 \\
\hline Negative & 14 & 42.43 \\
\hline Total & 33 & 100 \\
\hline * Unspecified cases were removed. \\
\hline
\end{tabular}

The mean score of GI symptoms in patients was 0.46 and its standard deviation was 0.27 .

Table 3 shows the distribution of positive cases of increased small intestine wall thickness in the CTEnterography of the subjects. According to this table, increased wall thickness was seen in 6 people $(16.7 \%)$ and in 30 people (83.3\%) it wasn't increased. Three people were not judged due to the lack of CTEnterography.

Table 3

Frequency distribution of positive cases of increased small intestine wall thickness

\begin{tabular}{|lll|}
\hline Increased small intestine wall thickness & Number & Percentage \\
\hline Positive & 6 & 16.70 \\
\hline Negative & 30 & 83.30 \\
\hline Total & 36 & 100 \\
\hline * Unspecified cases were removed. & & \\
\hline
\end{tabular}


Table 4 shows the distribution of positive cases of esophageal dilation according to the barium swallow test in the subjects studied. According to this table, 13 (36.1\%) patients had esophageal dilation and 23 (63.9\%) had no problem. Three patients were unable to do barium swallow test.

Table 4

Frequency distribution of positive cases of esophageal dilation, according to the barium swallow test in the subjects

\begin{tabular}{|lll|}
\hline \multicolumn{4}{|c|}{ Swallow test in the subjects } \\
\hline Positive & 13 & 36.10 \\
\hline Negative & 23 & 63.90 \\
\hline Total & 36 & 100 \\
\hline * Unspecified cases were removed. \\
\hline
\end{tabular}

Table 5 shows the mean and standard deviation of laboratory tests in the studied subjects. According to the table, the average and standard deviation for ESR, CRP, albumin, ferritin, folic acid, vitamin b12, magnesium, and zinc were 36.58 and $22.13,4.65$ and $7.12,4.03$ and $0.41,65.07$ and 82.38, 15.16 and $7.01,435.77$ and $1.86,2.06$ and $0.14,49.43$ and 28.94 respectively.

Table 5

The mean and standard deviation of laboratory tests in the subjects

\begin{tabular}{|lll|}
\hline Variable & Mean & Standard deviation \\
\hline ESR & 36.58 & 22.13 \\
\hline CRP & 4.65 & 7.12 \\
\hline Albumin & 4.03 & 0.41 \\
\hline Ferritin & 65.07 & 82.38 \\
\hline Folic acid & 15.16 & 7.01 \\
\hline Vitamin B12 & 435.77 & 1.86 \\
\hline Magnesium & 2.06 & 0.14 \\
\hline Zinc & 49.43 & 28.94 \\
\hline * Unspecified cases were removed.
\end{tabular}

In general, the FC test was successfully completed in 38 subjects. Out of these, the result of FC test was positive in $26(68.4 \%)$ patients and negative in $12(31.6 \%)$. 
Table 6 shows the distribution of FC results by age. According to this table, out of 17 patients in the young group, $\mathrm{FC}$ was negative in $5(29.4 \%)$ patients and was positive in $12(70.6 \%)$ patients and among 21 patients in the middle age group; FC was positive in 14 (66.7\%) and negative in 7 (33.3\%). The P value was 0.79 , indicating that the relationship between $\mathrm{FC}$ value and age group is not meaningful.

Table 6

The distribution of $\mathrm{FC}$ results by age in the subjects.

\begin{tabular}{|llllllll|}
\hline Age category & \multicolumn{2}{l}{ Negative FC } & \multicolumn{2}{l}{ Positive FC } & \multicolumn{2}{l}{ Total } & \multirow{2}{*}{ P Value } \\
\cline { 2 - 7 } & No. & $\%$ & No. & $\%$ & No. & $\%$ & \\
Young & 5 & 29.40 & 12 & 70.60 & 17 & 100 & 0.79 \\
Middle age & 7 & 33.30 & 14 & 66.70 & 21 & 100 & \\
\hline
\end{tabular}

Table 7 shows the distribution of FC results for different types of SSc. According to this table, out of 22 patients with diffuse disease, 6 (27.3\%) patients had negative FC responses and 16 (72.7\%) had positive FC responses. Among 14 patients with limited type of disease, FC response was negative in $5(35.7 \%)$ patients and was positive in $9(64.3 \%)$ cases. It was not possible to identify disease type in 2 patients, with present information. The $P$ value was 0.59 , which indicates that the relationship between the FC value and the type of disease is not significant.

Table 7

The distribution of FC results for different types of SSc in the subjects.

\begin{tabular}{|lllllllll|}
\hline \multirow{2}{*}{ Disease type } & \multicolumn{2}{l}{ Negative FC } & \multicolumn{2}{l}{ Positive FC } & \multicolumn{2}{l}{ Total } & P Value \\
\cline { 2 - 7 } & No. & $\%$ & No. & $\%$ & No. & $\%$ & \\
\cline { 1 - 7 } Diffused & 6 & 27.30 & 16 & 72.70 & 22 & 100 & 0.59 \\
\cline { 1 - 7 } Limited & 5 & 35.70 & 9 & 64.30 & 14 & 100 & \\
\hline
\end{tabular}

Table 8 shows the distribution of FC results according to the presence of wall thickening. Out of 30 patients with normal wall thickness, FC response was negative in $11(36.7 \%)$ patients and was positive in $19(63.3 \%)$ and every 5 patients with wall thickening had positive FC response. three patients didn't have CT-Enterography so small intestine wall thickening was not detectable. Considering the fact that $P$ value for the relation between FC value and wall thickness was 0.10 , this relationship was not statistically significant. 
Table 8

The distribution of FC results according to the presence of wall thickness in the subjects.

\begin{tabular}{|llllllll|}
\hline \multirow{2}{*}{ Increased small intestine wall thickness } & \multicolumn{2}{l}{ Negative FC } & \multicolumn{2}{l}{ Positive FC } & \multicolumn{2}{c|}{ Total } & \multicolumn{3}{c|}{ P Value } \\
\cline { 2 - 7 } & No. & $\%$ & No. & $\%$ & No. & $\%$ & \\
Diffused & 11 & 36.70 & 19 & 63.30 & 30 & 100 & 0.10 \\
Limited & 0 & 0.00 & 5 & 100 & 5 & 100 & \\
\hline
\end{tabular}

Table 9 shows the distribution of FC results according to the presence or absence of esophageal dilation in the subjects. Out of 22 patients without esophageal dilation, FC response was negative in $8(36.4 \%)$ patients and was positive in 14 (63.3\%) patients and in 13 patients with esophageal dilatation, FC response was negative in $2(15.4 \%)$ patients and was positive in $11(84.6 \%)$ patients. Three patients couldn't do barium swallow test, so esophageal dilation could not be determined in them. The $P$ value for the relationship between $\mathrm{FC}$ value and esophagus dilation was 0.18 , which indicates that this relationship is not significant.

Table 9

The distribution of FC results according to the presence or absence of esophageal dilation in the subjects.

\begin{tabular}{|llllllll|}
\hline \multirow{2}{*}{ Esophageal dilation } & \multicolumn{2}{l}{ Negative FC } & \multicolumn{2}{l}{ Positive FC } & \multicolumn{2}{l}{ Total } & \multirow{2}{*}{ P Value } \\
\cline { 2 - 7 } & No. & $\%$ & No. & $\%$ & No. & $\%$ & \\
Negative & 8 & 36.40 & 14 & 63.60 & 22 & 100 & 0.18 \\
\cline { 1 - 7 } Positive & 2 & 15.40 & 11 & 84.60 & 13 & 100 & \\
\hline
\end{tabular}

Table 10 shows the mean and standard deviation of skin involvement score and the final score for $\mathrm{GI}$ symptoms and ESR and magnesium in terms of both positive and negative FC. According to this table, the mean and standard deviation of skin involvement score in subjects with negative FC response were 11.38 and 7, respectively, and these values were 15.92 and 10.08 , respectively, in subjects with positive $F C$ responses $(P$. Value $=0.25)$. The mean and standard deviation of gastrointestinal symptoms in subjects with negative FC response were 0.39 and 0.24 , respectively, and these values were 0.49 and 0.28 , respectively, in subjects with positive FC response ( $p$ value $=0.30)$. The mean and standard deviation of ESR in subjects with negative FC response were 0.39 and 22.96, respectively, and these values were 35.46 and 22.11, respectively, in subjects with positive $F C$ responses $(P$. Value $=0.65)$. Mean and standard deviation of magnesium in subjects with negative FC response were 2.06 and 0.07 , respectively, and these values were 2.06 and 0.16 , respectively, in subjects with positive $F C$ responses $(P$. Value $=0.94)$. The calculated P. Values showed that the relationship of FC with any of these variables was not significant. 
Table 10

The mean and standard deviation of skin involvement score and the final score for GI symptoms and ESR and Magnesium in terms of both positive and negative FC.

\begin{tabular}{|c|c|c|c|c|c|}
\hline \multirow[t]{2}{*}{ Variable } & \multicolumn{2}{|c|}{ Negative FC } & \multicolumn{2}{|c|}{ Positive FC } & \multirow[t]{2}{*}{ P Value $^{*}$} \\
\hline & Mean & SD & Mean & SD & \\
\hline Skin score & 11.38 & 7.00 & 15.92 & 10.08 & 0.25 \\
\hline Digestive score & 0.39 & 0.24 & 0.49 & 0.28 & 0.30 \\
\hline ESR & 39 & 22.96 & 35.46 & 22.11 & 0.65 \\
\hline Magnesium & 2.06 & 0.07 & 2.06 & 0.16 & 0.94 \\
\hline
\end{tabular}

Table 11 shows the mean and standard error of serum CRP, albumin, ferritin, folic acid, vitamin B12, and zinc, in terms of both positive and negative FC. According to this table, mean and standard error of CRP in subjects with negative FC response were 3.32 and 1.78, respectively, and these values were 5.26 and 1.48 , respectively, in subjects with positive $F C$-response $(P$. Value $=0.21)$. The mean and standard error of albumin in subjects with negative $\mathrm{FC}$ response were 3.92 and 0.11 , respectively, and these values in patients with positive FC responses were 4.07 and 0.08 respectively $(P$. Value $=0.22)$. The mean and standard error of ferritin in subjects with negative FC response was 73.32 and 19.56 , respectively, and these values in subjects with positive FC response were 61.26 and 17.51, respectively (P. Value $=0.10)$. The mean and standard error in subjects with a negative FC response was 12.06 and 2.02, respectively, and these values in subjects with positive FC-response were 16.33 and 1.33 , respectively ( $p$ value $=0.11$ ). Mean and standard error of vitamin B12 in subjects with negative FC response were 453.85 and 40.23 , respectively, and these values in subjects with positive FC response were 427.41 and 40.34 , respectively $(P$. Value $=0.45)$. Mean and standard deviation of Zinc in patients with negative FC response were 44.75 and 3.08, respectively, and these values in subjects with positive FC-response were 51.58 and 6.71 (P. Value $=0.84)$. Due to the values calculated as $P$. Value for the correlation of each variable with $F C$, there is no significant relationship between them. 
Table 11

The mean and error of serum CRP, albumin, ferritin, folic acid, vitamin B12, and zinc, in terms of both positive and negative FC.

\begin{tabular}{|c|c|c|c|c|c|}
\hline \multirow[t]{2}{*}{ Variable } & \multicolumn{2}{|c|}{ Negative FC } & \multicolumn{2}{|c|}{ Positive FC } & \multirow[t]{2}{*}{ P Value } \\
\hline & Mean & SE & Mean & SE & \\
\hline CRP & 3.32 & 1.78 & 5.26 & 1.48 & 0.21 \\
\hline Albumin & 3.94 & 0.11 & 4.07 & 0.08 & 0.22 \\
\hline Ferritin & 73.32 & 19.56 & 61.26 & 17.51 & 0.10 \\
\hline Folic acid & 12.60 & 2.02 & 16.33 & 1.33 & 0.11 \\
\hline Vitamin B12 & 453.85 & 40.23 & 427.41 & 40.34 & 0.45 \\
\hline Zinc & 44.75 & 3.08 & 51.58 & 6.71 & 0.84 \\
\hline
\end{tabular}

\section{Discussion}

With regards to determination of FC positive cases by age, as it is shown in Table 6, there wasn't a significant difference between the frequencies of FC positive cases in the young group $(70.6 \%)$ and middle-aged group $(66.7 \%)(P$. Value $=0.79)$. However, this comparison suggests a higher frequency of positive FC cases in terms of numerical value in the younger age group, which can be attributed to the higher activity of digestive tract in younger people. In a study (40), the mean FC level in elderly patients with IBD that had normal endoscopic findings was not higher and there was no significant relationship between age and level of stool calprotectin. Another study by D'Angelo in 2017 showed that FC levels increase with age (41).

In our study, the determination of the frequency of FC cases by gender was not feasible, because out of 46 patients in this study, only 2 were male and their information has not entered our surveys from the beginning, due to the exit criteria. So, all patients in our study were of female and they could not be compared by gender.

About the frequency of positive cases of FC in patients with systemic sclerosis by disease type, according to the information in Table 7, between the frequency of FC positive cases in the diffused type $(72.7 \%)$ and the frequency of FC positive cases in the limited type (64.3\%) wasn't a statistically significant difference (P. Value $=0.59)$. However, as expected, and due to the higher probability of digestive tract disorder in the diffuse type (42), the number of positive FCs in the diffuse type was significantly higher than the limited type of disease which confirms the link between FC levels and digestive tract involvement in patients with systemic sclerosis. While Bonnin et al (43) also conducted a study in 2007 to evaluate the use and sensitivity of FC in children with signs and symptoms of IBD and the results of this study indicated that the level of FC in children with IBD is significantly higher than that in children with functional type of 
disease or healthy children. Also, Andreasson et al (11) in 2014, conducted a study to evaluate the specificity of FC for diagnosing the digestive tract problems in SSc patients, the researchers found that the level of FC in SSc patients was significantly higher compared to people with Sjögren's disease, rheumatoid arthritis, and healthy ones. Ozseker et al (44), also in 2016 had a study about determining the role of FC to show digestive tract disorders in patients with behcet's disease, patients with ulcers in terminal ileum or colon in endoscopic examinations, had significantly higher levels of FC compared to patients with no digestive tract abnormalities $(P$. Value $=0.01)$. At the end of the study, the researchers concluded that determining FC levels could be used as a good tool for identifying gastrointestinal tract problems in patients with behcet's disease who do not have gastrointestinal symptoms. Contrary to the above studies, Montalto et al (24), in 2013, reviewed the results of recent studies on the diagnostic use of FC levels in various gastrointestinal disorders. The researchers concluded that FC level should not be used as a marker for structural digestive tract diseases, Instead, it can only be described as a sign of inflammation of the gastrointestinal wall. As previously mentioned, these results contradicted the results of other studies.

About the determination of the prevalence of FC positive cases in patients with systemic sclerosis, by the thickness of the intestinal wall in CT-enterography, according to the results that can be seen in Table 8, There was no statistically significant difference between the frequency of FC positive cases in people with normal small intestine wall thickness (63.3\%) and those with increased small intestine wall thickness $(100 \%)(P$. Value $=0.10)$. it can be explained by the fact that only 5 cases had evidence of increased small intestinal wall thickness in their CT-enterography and all those 5, had FC levels of more than $200 \mathrm{~g} / \mathrm{g} \mu$. So due to the fact that endoscopic access to the small intestine is difficult, expensive and painful, the determination of FC level can be used as a predictive tool for the presence of gastrointestinal disorder in the small intestine in patients with SSc. In two studies conducted by Anderson et al. (2011) (2) and 2014 (11), a correlation between FC level and gastrointestinal pathology which was proved by Cineradiography. In another study, Cerrillo et al. (45), in 2015, did a magnetic resonance imaging (MRE) test for 112 patients with ileum involved Crohn's disease and compared the results of this test with FC levels in these patients. The Magnetic Resonance Index of Activity score was significantly associated with FC levels ( $p$ value $<0.01$ ). Also, in a study by Shimoyama et al. (46) in 2017 to evaluate FC levels as a marker for small bowel inflammation in patients with Crohn's disease, there was a significant positive correlation between FC level and CT-enterography score $(P<0.0001)$. these studies were congruent with our results. The reason why this correlation was not meaningful in our study can be the low sample size which results in the low number of people with evidence of impaired CT-enterography. We hope that in future studies, by assigning a larger sample size, the role of $\mathrm{FC}$ as a sign of inflammation in the small intestine becomes more intense.

Esophagus is the most common part of the gastrointestinal tract which is affected in patients with systemic sclerosis and is commonly involved in $90 \%$ of patients (47). To compare the frequency of FC positive cases in the presence or absence of esophageal dilation in the barium swallow test, the information obtained from our study is presented in Table 9. According to the results, there was no statistically significant relationship between the frequency of positive cases of FC in patients without 
esophageal dilation (63.6\%) and those with esophageal dilation (84.6\%) (P. Value $=0.18)$. However, according to the results, FC levels in those with esophageal dilation were numerically higher than levels of FC in patients with no esophageal dilation, which could indicate a correlation between elevation of FC level and upper gastrointestinal tract disorders. Manz et al in 2012 (40) evaluated FC levels as a marker in those who referred to the clinics because of abdominal pain. They concluded that FC was in fact a parameter that could be used to detect abnormalities in both upper and lower gastrointestinal tract. But, according to these researchers, the diagnostic value of FC is more in colon disorders. Also, in a study by Canani et al. in 2004 (48) to evaluate the relationship between the diagnostic value of FC and clinical symptoms of digestive problems in children, The researchers concluded that the mean FC level in children with reflux was $138 \mu \mathrm{g} / \mathrm{g}$ and in healthy children was $28 \mu \mathrm{g} / \mathrm{g}$, this difference was statistically significant ( $p$ value $<0.001$ ), and it indicated the relationship between FC level and upper gastrointestinal disorders.

According to Table 10, we found that the average skin score in those with positive FC response was 15.92 , and in those with negative FC response was 11.38 . The difference was not statistically significant (P. Value $=0.25$ ). In confirmation of this result, Andreasson et al. (2) also conducted a study in 2011 about the role of $\mathrm{FC}$ as a biomarker for digestive tract disorders in systemic sclerosis patients and there was not a significant correlation between FC level and skin score obtained by MRSS, accordingly, in the following studies, they did not compare these two parameters. In addition, in a study by Marie et al. (1) in 2015 about the role of $\mathrm{FC}$ level in various aspects of systemic sclerosis, they could not find a correlation between the FC level and the average skin score $(P$. Value $=0.79)$.

About the FC level and gastrointestinal symptoms score, as shown in Table 10, the average gastrointestinal symptoms score in those with positive $\mathrm{FC}$ response was 0.49 , while it was 0.39 in those with negative FC response. This indicates that the association between FC and gastrointestinal symptoms is not statistically significant $(P$. Value $=0.30)$. One reason could be the lack of specialization of the questionnaire for Iranian people. The use of anti-acid drugs, such as proton pump inhibitors (PPIs), could be another reason for this claim. On the other hand, it is important to note that one part of the questionnaire is about fear of digestive problems when confronted with various social conditions, while most of our patients were middle-aged, illiterate, and housewife women; consequently, they have not confronted different social conditions. In another part of the questionnaire, there was a question about the fear of digestive problems in traveling, while most of our patients could not travel because of financial problems or being bedridden because of their physical problems, therefore, it was not possible to examine such parts of the questionnaire. Additionally, Andreasson et al. in 2011(2), studying the role of FC as a biomarker in digestive disorders in patients with systemic sclerosis, concluded that the relation between FC level and the gastrointestinal symptom score obtained from a Swedish questionnaire (GSRS) is not statistically significant $(P$. Value $=0.44)$. They also explained this non-significant relationship, as the questionnaire was not specialized for systemic sclerosis patients.

Malnutrition is known as an important symptom of acute gastrointestinal disease (49). The number of bacteria in the small intestine, which is the main site of nutrients absorption, is low (50). SIBO can cause 
food to be consumed by bacteria and also damage the mucosa, thereby impairing absorption in the small intestine and causing malabsorption (51). Finding out about micronutrient deficiency which is one of malnutrition symptoms, can be obtained by various laboratory tests (52). In Polkowska-Pruszyńskas's study, FC is a sensitive and specific indicator for the diagnosis of SIBO in SSc patients, but FC alone is not sufficient to diagnose SIBO (53). the Calprotectin level correlated with intestinal wall thickness, the presence of (SIBO), and micronutrients of studied patients. the study concluded that SIBO treatment for more than 3 months could affect fecal Calprotectin (15). Tables 10 and 11 show the mean serum tests (ESR, magnesium, CRP, albumin, ferritin, folic acid, vitamin B12 and zinc) in two groups of patients with positive $\mathrm{FC}$ response and negative $\mathrm{FC}$ response and none of these cases had a statistically significant relationship with FC level (P. Value $\geq 0.10$ ). Even contrary to our expectations, the albumin level in the positive FC group (4.07) was more than the negative FC group (3.94). Also, about folic acid, the mean of FC-positive group was 16.33 and in the negative FC group was 12.6, or about zinc, the mean of FC positive group was 51.58 but the mean of negative FC group was 44.75. Meanwhile, we expected that the levels of micronutrients in the FC-positive group would be lower due to more gastrointestinal involvement and higher malabsorption also immobility of the small intestine increases the growth of bacteria that occurs as result of malabsorption.

We conclude that one of the most important reasons for the high levels of these micronutrients in the FCpositive group was the receipt of various vitamin and mineral supplements as prescribed by the physician for these patients. The study by Andreasson et al. (2011) (2) also confirmed the results of our study. The researchers found that FC levels in patients who received micronutrient supplements due to micronutrient deficiencies were higher than patients who had not received any supplemental medications. In another study by the same researchers in 2014 (11), levels of folic acid, vitamin B12, iron, and zinc were measured in SSc patients. The FC level in people with a deficiency of more than one micronutrient was higher than those with just one micronutrient deficiency. Also, FC in the second group was higher than normal subjects (P. Value $=0.001)$, which itself indicates that gastrointestinal disorders in SSc patients is followed by an increase in FC levels.

\section{Conclusion}

According to the results of this study, there was no statistically significant relationship between FC levels of higher than $200 \mu \mathrm{g} / \mathrm{g}$ and all of the variables such as age, sex, type of disease, increased thickness of small bowel wall, esophageal dilation, gastrointestinal complaints, skin score and micronutrient deficiencies. However, there was a numerically significant correlation between the FC levels of more than $200 \mu \mathrm{g} / \mathrm{g}$ and the increase in the thickness of the small bowel wall and esophageal dilation. This could indicate the proper use of FC as a marker for the diagnosis of gastrointestinal tract involvement in patients with systemic sclerosis. Using this method, in addition to reducing the costs, is capable of preventing invasive and time-consuming methods such as endoscopy. It means that if the patient with systemic sclerosis has FC levels more than $200 \mu \mathrm{g} / \mathrm{g}$, it is advisable for the patient to continue the examination using another method, such as endoscopy. Additionally, most patients use various vitamin and mineral supplements to prevent malnutrition. Consequently, there was no micronutrient deficiency 
among patients. The main cause of the lack of statistical significance in the relationships of our study can be the low sample size. Therefore, it is recommended that in future researchers' study with a larger sample size.

\section{Declarations}

Ethical approval and consent to participate: The study and all experimental protocols were approved by the Professional Ethics Committee of the Golestan University of Medical Sciences with the ethical code of ir.goums.rec.1396.84. The informed consent was also obtained from all patients for the implementation of this project.

All methods were carried out in accordance with relevant guidelines and regulations.

Consent for publication: Consent has been obtained from all patients to participate in the study and dissemination of their information in accordance with the principles of confidentiality.

Availability of data and materials: The data that support the findings of this study are available from Nafiseh Abdolahi but restrictions apply to the availability of these data, which were used under license for the current study, and so are not publicly available. Data are however available in the form of an Excel file from the authors upon reasonable request and with permission of Nafiseh Abdolahi.

Competing interests: The authors declare no competing interest.

Funding: The Golestan University of Medical Sciences financially supported this work.

Role of the Funder/Sponser: The funder had no role in the design and conduct of the study; collection, management, analysis, and interpretation of the data; preparation, review, or approval of the manuscript; and decision to submit the manuscript for publication.

\section{Authors contribution:}

A.F performed data entry and writing article. R.Z performed writing article. H.H performed visiting patients and data collection. N.A and M.AGH performed data collection and article review. H.GH and S.L performed radiologic analysis and interpretation.

Acknowledgment: Special thanks to all the fellow authors who contributed to this article.

\section{References}

1. Marie I, Leroi AM, Menard JF, Levesque H, Quillard M, Ducrotte P. Fecal calprotectin in systemic sclerosis and review of the literature. Autoimmunity reviews. 2015;14(6):547-54.

2. Andreasson K, Scheja A, Saxne T, Ohlsson B, Hesselstrand R. Faecal calprotectin: a biomarker of gastrointestinal disease in systemic sclerosis. J Intern Med. 2011;270(1):50-7. 
3. Abraham DJ, Varga J. Scleroderma: from cell and molecular mechanisms to disease models. Trends in immunology. 2005;26(11):587-95.

4. Varga J, Abraham D. Systemic sclerosis: a prototypic multisystem fibrotic disorder. The Journal of Clinical Investigation. 2007;117(3):557-67.

5. Affandi AJ, Radstake TR, Marut W. Update on biomarkers in systemic sclerosis: tools for diagnosis and treatment. Seminars in immunopathology. 2015;37(5):475-87.

6. Domsic R, Fasanella K, Bielefeldt K. Gastrointestinal manifestations of systemic sclerosis. Digestive diseases and sciences. 2008;53(5):1163-74.

7. LeRoy EC, Medsger TA, Jr. Criteria for the classification of early systemic sclerosis. The Journal of rheumatology. 2001;28(7):1573-6.

8. Krishnan E, Furst DE. Systemic sclerosis mortality in the United States: 1979-1998. European journal of epidemiology. 2005;20(10):855-61.

9. Maricq HR, Weinrich MC, Keil JE, Smith EA, Harper FE, Nussbaum Al, et al. Prevalence of scleroderma spectrum disorders in the general population of South Carolina. Arthritis and rheumatism. 1989;32(8):998-1006.

10. Arnett FC, Cho M, Chatterjee S, Aguilar MB, Reveille JD, Mayes MD. Familial occurrence frequencies and relative risks for systemic sclerosis (scleroderma) in three United States cohorts. Arthritis and rheumatism. 2001;44(6):1359-62.

11. Andreasson K, Saxne T, Scheja A, Bartosik I, Mandl T, Hesselstrand R. Faecal levels of calprotectin in systemic sclerosis are stable over time and are higher compared to primary Sjogren's syndrome and rheumatoid arthritis. Arthritis research \& therapy. 2014;16(1):R46.

12. Forbes A, Marie I. Gastrointestinal complications: the most frequent internal complications of systemic sclerosis. Rheumatology (Oxford, England). 2009;48 Suppl 3:iii36-9.

13. Nagaraja V, McMahan ZH, Getzug T, Khanna D. Management of gastrointestinal involvement in scleroderma. Current treatment options in rheumatology. 2015;1(1):82-105.

14. Hesselstrand R, Scheja A, Akesson A. Mortality and causes of death in a Swedish series of systemic sclerosis patients. Annals of the rheumatic diseases. 1998;57(11):682-6.

15. Ebrahimi B, Nazarinia M, Molayem M. Calprotectin, an available prognostic biomarker in systemic sclerosis: a systematic review. Clin Rheumatol. 2021;40(5):1709-15.

16. Alastal Y, Hammad TA, Renno A, Khalil B, Pierre J, Kwaah B, et al. Gastrointestinal manifestations associated with systemic sclerosis: results from the nationwide inpatient sample. Ann Gastroenterol. 2017;30(5):498-503.

17. Villalta D, Imbastaro T, Di Giovanni S, Lauriti C, Gabini M, Turi MC, et al. Diagnostic accuracy and predictive value of extended autoantibody profile in systemic sclerosis. Autoimmunity reviews. 2012;12(2):114-20.

18. Marie I, Ducrotte P, Antonietti M, Herve S, Levesque H. Watermelon stomach in systemic sclerosis: its incidence and management. Alimentary pharmacology \& therapeutics. 2008;28(4):412-21. 
19. Marie I, Gourcerol G, Leroi AM, Menard JF, Levesque H, Ducrotte P. Delayed gastric emptying determined using the 13C-octanoic acid breath test in patients with systemic sclerosis. Arthritis and rheumatism. 2012;64(7):2346-55.

20. Elhai M, Avouac J, Kahan A, Allanore Y. Systemic sclerosis at the crossroad of polyautoimmunity. Autoimmunity reviews. 2013;12(11):1052-7.

21. Manetti M, Neumann E, Muller A, Schmeiser T, Saar P, Milia AF, et al. Endothelial/lymphocyte activation leads to prominent CD4 + T cell infiltration in the gastric mucosa of patients with systemic sclerosis. Arthritis and rheumatism. 2008;58(9):2866-73.

22. Ayling RM, Kok K. Fecal Calprotectin. Adv Clin Chem. 2018;87:161-90.

23. Costa F, Mumolo MG, Bellini M, Romano MR, Ceccarelli L, Arpe P, et al. Role of faecal calprotectin as non-invasive marker of intestinal inflammation. Digestive and liver disease: official journal of the Italian Society of Gastroenterology and the Italian Association for the Study of the Liver. 2003;35(9):642-7.

24. Montalto M, Gallo A, Santoro L, D'Onofrio F, Landolfi R, Gasbarrini A. Role of fecal calprotectin in gastrointestinal disorders. European review for medical and pharmacological sciences. 2013;17(12):1569-82.

25. Johne B, Fagerhol MK, Lyberg T, Prydz H, Brandtzaeg P, Naess-Andresen CF, et al. Functional and clinical aspects of the myelomonocyte protein calprotectin. Molecular Pathology. 1997;50(3):11323.

26. Bjerke K, Halstensen TS, Jahnsen F, Pulford K, Brandtzaeg P. Distribution of macrophages and granulocytes expressing L1 protein (calprotectin) in human Peyer's patches compared with normal ileal lamina propria and mesenteric lymph nodes. Gut. 1993;34(10):1357-63.

27. Vermeire S, Van Assche G, Rutgeerts P. Laboratory markers in IBD: useful, magic, or unnecessary toys? Gut. 2006;55(3):426-31.

28. Roseth AG, Fagerhol MK, Aadland E, Schjonsby H. Assessment of the neutrophil dominating protein calprotectin in feces. A methodologic study. Scandinavian journal of gastroenterology. 1992;27(9):793-8.

29. Gisbert JP, McNicholl AG. Questions and answers on the role of faecal calprotectin as a biological marker in inflammatory bowel disease. Digestive and liver disease: official journal of the Italian Society of Gastroenterology and the Italian Association for the Study of the Liver. 2009;41(1):5666.

30. Angriman I, Scarpa M, D'Inca R, Basso D, Ruffolo C, Polese L, et al. Enzymes in feces: useful markers of chronic inflammatory bowel disease. Clinica chimica acta; international journal of clinical chemistry. 2007;381(1):63-8.

31. Konikoff MR, Denson LA. Role of fecal calprotectin as a biomarker of intestinal inflammation in inflammatory bowel disease. Inflammatory bowel diseases. 2006;12(6):524-34.

32. Johnson MW, Maestranzi S, Duffy AM, Dewar DH, Forbes A, Bjarnason I, et al. Faecal calprotectin: a noninvasive diagnostic tool and marker of severity in pouchitis. European journal of gastroenterology \& hepatology. 2008;20(3):174-9. 
33. Urade M, Shinbo T. Barium Appendicitis 1 Month After a Barium Meal. International Surgery. 2012;97(4):296-8.

34. Cakmakci E, Erturk SM, Cakmakci S, Bayram A, Tokgoz S, Caliskan KC, et al. Comparison of the results of computerized tomographic and diffusion-weighted magnetic resonance imaging techniques in inflammatory bowel diseases. Quantitative imaging in medicine and surgery. 2013;3(6):327-33.

35. van den Hoogen F, Khanna D, Fransen J, Johnson SR, Baron M, Tyndall A, et al. 2013 classification criteria for systemic sclerosis: an American college of rheumatology/European league against rheumatism collaborative initiative. Annals of the rheumatic diseases. 2013;72(11):1747-55.

36. Kaldas M, Khanna PP, Furst DE, Clements PJ, Kee Wong W, Seibold JR, et al. Sensitivity to change of the modified Rodnan skin score in diffuse systemic sclerosis-assessment of individual body sites in two large randomized controlled trials. Rheumatology (Oxford, England). 2009;48(9):1143-6.

37. Valentini G, D’Angelo S, Della Rossa A, Bencivelli W, Bombardieri S. European Scleroderma Study Group to define disease activity criteria for systemic sclerosis. IV. Assessment of skin thickening by modified Rodnan skin score. Annals of the rheumatic diseases. 2003;62(9):904.

38. Khanna D, Hays RD, Maranian P, Seibold JR, Impens A, Mayes MD, et al. Reliability and validity of UCLA Scleroderma Clinical Trial Consortium Gastrointestinal Tract (UCLA SCTC GIT 2.0) Instrument. Arthritis and rheumatism. 2009;61(9):1257-63.

39. Moum B, Jahnsen J, Bernklev T. Fecal calprotectin variability in Crohn's disease. Inflamm Bowel Dis. 2010;16(7):1091-2.

40. Manz M, Burri E, Rothen C, Tchanguizi N, Niederberger C, Rossi L, et al. Value of fecal calprotectin in the evaluation of patients with abdominal discomfort: an observational study. BMC gastroenterology. 2012;12:5.

41. D'Angelo F, Felley C, Frossard JL. Calprotectin in Daily Practice: Where Do We Stand in 2017 ? Digestion. 2017;95(4):293-301.

42. Carwile LeRoy E, M. Black C, Fleischmajer R, Jablonska S, Tr K, Medsger JT, et al. Scleroderma (Systemic Sclerosis): Classification, Subsets and Pathogenesis1988. 202-5 p.

43. Bonnin Tomas A, Vila Vidal M, Rosell Camps A. [Fecal calprotectin as a biomarker to distinguish between organic and functional gastrointestinal disease]. Revista espanola de enfermedades digestivas: organo oficial de la Sociedad Espanola de Patologia Digestiva. 2007;99(12):689-93.

44. Ozseker B, Sahin C, Ozseker HS, Efe SC, Kav T, Bayraktar Y. The Role of Fecal Calprotectin in Evaluating Intestinal Involvement of Behcet's Disease. Disease markers. 2016;2016:5423043.

45. Cerrillo E, Beltran B, Pous S, Echarri A, Gallego JC, Iborra M, et al. Fecal Calprotectin in Ileal Crohn's Disease: Relationship with Magnetic Resonance Enterography and a Pathology Score. Inflammatory bowel diseases. 2015;21(7):1572-9.

46. Shimoyama T, Yamamoto T, Umegae S, Matsumoto K. Faecal biomarkers for screening small bowel inflammation in patients with Crohn's disease: a prospective study. Therapeutic Advances in Gastroenterology. 2017;10(8):577-87. 
47. Rose S, Young MA, Reynolds JC. GASTROINTESTINAL MANIFESTATIONS OF SCLERODERMA. Gastroenterology Clinics of North America. 1998;27(3):563-94.

48. Berni Canani R, Rapacciuolo L, Romano MT, Tanturri de Horatio L, Terrin G, Manguso F, et al. Diagnostic value of faecal calprotectin in paediatric gastroenterology clinical practice. Digestive and Liver Disease. 2004;36(7):467-70.

49. Medsger TA, Jr., Silman AJ, Steen VD, Black CM, Akesson A, Bacon PA, et al. A disease severity scale for systemic sclerosis: development and testing. The Journal of rheumatology. 1999;26(10):215967

50. Parrish C, Zaidel O, Lin HC, editors. Uninvited Guests: The Impact of Small Intestinal Bacterial Overgrowth on Nutritional Status NUTRITION ISSUES IN GASTROENTEROLOGY, SERIES \#72003.

51. McFarlane IM, Bhamra MS, Kreps A, lqbal S, Al-Ani F, Saladini-Aponte C, et al. Gastrointestinal Manifestations of Systemic Sclerosis. Rheumatology (Sunnyvale). 2018;8(1):235.

52. Clements PJ, Becvar R, Drosos AA, Ghattas L, Gabrielli A. Assessment of gastrointestinal involvement. Clinical and experimental rheumatology. 2003;21(3 Suppl 29):S15-8.

53. Polkowska-Pruszyńska B, Gerkowicz A, Rawicz-Pruszyński K, Krasowska D. The Role of Fecal Calprotectin in Patients with Systemic Sclerosis and Small Intestinal Bacterial Overgrowth (SIBO). Diagnostics (Basel). 2020;10(8):587. 